\title{
Mehrsprachige Jugendliche im Umgang mit Dialekt und Hochsprache in der Deutschen Schweiz
}

\author{
Annelies Häcki Buhofer/Hansjakob Schneider/Christine Beckert (Basel/Aarau)
}

\begin{abstract}
For much too long the study of plurilingual pupils has been focussed on their deficits. In the present study special resources of underprivileged adolescents who are successful in their literal socialization were investigated. From a sample of 1500 15-year-olds 76 were asked to fulfil a writing task measuring their functional-pragmatic writing abilities. The results show only minor differences between mono- and plurilingual adolescents.

The same trend can be detected for reading abilities as well, especially when empathic reading competence is included and socio-economic status is controlled for.

The authors argue in favour of a concept for reading and writing which, in addition to traditional hard criteria like orthography and grammar for writing or information processing for reading, incorporates soft dimensions like emotional involvement (for reading) or communicative strategies (for writing). Reading and writing socialization viewed in such terms can only succeed if schools find ways to embed reading and writing in their students' lives in a way which is perceived by them as meaningful.
\end{abstract}

\section{$1 \quad$ Fragestellung und Begründung}

Das Konzept der Resilienz wird - vorwiegend in der Erziehungswissenschaft und Psychologie - verwendet, um von der Perspektive der unterstellten Defizienz wegzukommen. Statt zu fragen, welche Gruppen von Kindern schlechte Resultate erreichen und diesen Resultaten in anwendungsorientierten Studien soziale Fördermaßnahmen gegenüberzustellen, werden Entwicklungsverläufe in den Blick genommen, die bei einem Entwicklungsrisiko also trotz ungünstiger Konstellation - gute Resultate zeigen. Diese sind damit durch Resilienz gekennzeichnet, einer Situation also, in der positive Faktoren die Risikofaktoren ausgleichen (können). Unser Projekt zur "Literalen Resilienz" fragt in breiter und daher interdisziplinärer positiver Ausrichtung danach, was Jugendlichen aus bildungsfernen Elternhäusern bei der Bewältigung der Lese- und Schreibentwicklung hilft.

Der Weg zum Erwerb einer mehrsprachigen Kompetenz wurde und wird immer wieder als Risikofaktor der Entwicklung gehandelt. Mehrfacher Spracherwerb gilt als Phänomen, das bei Kindern und Jugendlichen eine Überbelastung darstellen kann ("surcharge cognitive"; cf. Lüdi 1986, 1999, 2007), die es - im Falle des Fremdsprachunterrichts als schulpolitische Aufgabe - zu dosieren gilt. Im Falle von Deutsch als Zweitspracherwerb ist eine Familien-

\footnotetext{
* Die Mitarbeitenden des SNF-Projekts "Literale Kompetenzen und literale Sozialisation von Jugendlichen aus schriftfernen Lebenswelten - Faktoren der Resilienz oder: Wenn Schriftaneignung trotzdem gelingt" sind: Erziehungswissenschaft: Wassilis Kassis, FHNW, Uni Basel, Ursula Stalder, FHNW, Uni Basel, Winfried Kronig, PH Bern, Uni Fribourg); Leseforschung: Andrea Bertschi-Kaufmann, FHNW, Esther Wiesner, FHNW, Hansjakob Schneider, FHNW; Schreibforschung: Annelies Häcki Buhofer, Uni Basel, Christine Beckert, Uni Basel.
} 
und Lebenssituation mit mehr als einer Sprache "unausweichlich" gegeben und nicht durch einen schulpolitischen Entscheid vorgesehen. Welche Konstellationen mehrsprachlichen Jugendlichen beim Ausbau ihrer literalen Kompetenzen entgegen kommen und sie vor einer ungenügenden Entwicklung schützen, ist die zentrale Frage unseres Projektes, zu der wir hier allererste Ergebnisse präsentieren, die wir zum "Konstrukt des leistungsschwachen Immigrantenkindes" (Kronig 2003) in Beziehung setzen möchten.

Dabei prüft unsere Analyse die in der Deutschen Schweiz immer wieder interessierende Frage, ob die diglossische Sprachsituation eine zusätzliche Schwierigkeit für den Erwerb des Deutschen als Zweitsprache darstellt bzw. ob eine solche Komplexität der Situation im Jugendalter allenfalls negative Auswirkungen hat. Die aktuelle Kindergarten- und Schulpolitik hat beispielsweise in den Kantonen Zürich und Basel seit einigen Jahren in Schulen mit großem Fremdsprachigen-Anteil Schulversuche initiiert, in denen in den stark dialektgeprägten ersten Kindergarten- und Schuljahren der Versuch einer nicht-diglossischen, sondern ausschließlich hochdeutschen Schul-Kommunikation gemacht wird. Hierbei handelt es sich um eine Aufgabe, auf die auch die Lehrkräfte entsprechend vorbereitet werden müssen, weil hochdeutsche Alltagskommunikation in der Schule nicht nur eine Frage der Kenntnisse, sondern auch der Häufigkeit des Sprachgebrauchs darstellt. Diese Schulversuche werden wissenschaftlich begleitet (cf. Gyger 2005, 2006 und Landert 2007).

\section{Datengrundlage}

Unsere Studie untersucht ca. 1500 Jugendliche im 8. und 9. Schuljahr in Deutschschweizer Schulen aller Leistungsansprüche. Sie kann also allgemeine und statistisch gut gestützte Aussagen zum Stand der Lese- und Schreibkompetenzen ebenso wie der Lese- und Schreibtätigkeiten der Jugendlichen machen. Da die Datenerhebung keine Bedingungen bezüglich der Muttersprache der ProbandInnen vorsah, wurden auch die Daten der mehrsprachigen Kinder in den untersuchten Klassen erfasst, die mit einer nichtdeutschen Muttersprache im Elternhaus in der deutschen Schweiz zur Schule gehen.

Von 1503 Kindern sind 467 mehrsprachig. Ihre Mehrsprachigkeit ist anhand der Daten des schriftlichen Fragebogens bestimmt worden, der Fragen nach der Sprache der Familie, des Vaters, der Mutter, der Kommunikation mit KollegInnen, der Förderung in verschiedenen Sprachen etc. enthält.

Wichtig ist, dass wir im Folgenden unter mehrsprachigen Jugendlichen junge Menschen verstehen, die als Erstsprache eine andere Sprache als Deutsch gelernt haben. In diese Definition nicht eingeschlossen sind Jugendliche, die eine Varietät des Deutschen als Erstsprache gelernt haben und sich durch Fremdsprachunterricht in Richtung funktionaler Mehrsprachigkeit bewegen. Wer sich selbst als mehrsprachig - mit nichtdeutscher Erstsprache - bezeichnet hat, wurde ungeachtet der konkreten Qualitäten der jeweiligen Fremdsprachkenntnisse entsprechend kategorisiert; auch weitere Faktoren wie "Unterricht in Deutsch für Fremdsprachige", "Sprachwahl in der Peergroup" etc. blieben dabei außer Acht. Allerdings bezeichnen wir in unserer Studie Jugendliche nur dann als mehrsprachig, wenn sie angeben, die Erstsprache in ihrem Alltag auch zu verwenden. Die Gruppe der mehrsprachigen Kinder ist also heterogen, wie es durch Sprachkompetenzen und Sprachgebrauch definierte Gruppen in vielen Fällen sind. So können beide Eltern oder nur ein Elternteil "nichtdeutschsprachig" sein, es kann eine "nicht-deutsche" Familiensprache (mit entsprechendem Freifachunterricht in der Schule) oder deutscher/dialektale Familiensprache, jedoch "nichtdeutscher" Erstsprache vorliegen.

Zusätzlich zu den Fragebogendaten sind weitere Daten durch Teilstichproben erhoben worden, die je nach Aufwändigkeit der Erhebung und Möglichkeiten der Auswertung als geringere Teilmengen festgelegt werden mussten. Sie umfassen folgende Erhebungen, die 
hier nicht näher charakterisiert werden können. ${ }^{1}$ Die Daten werden in kombinierten quantitativen und qualitativen Verfahren analysiert.

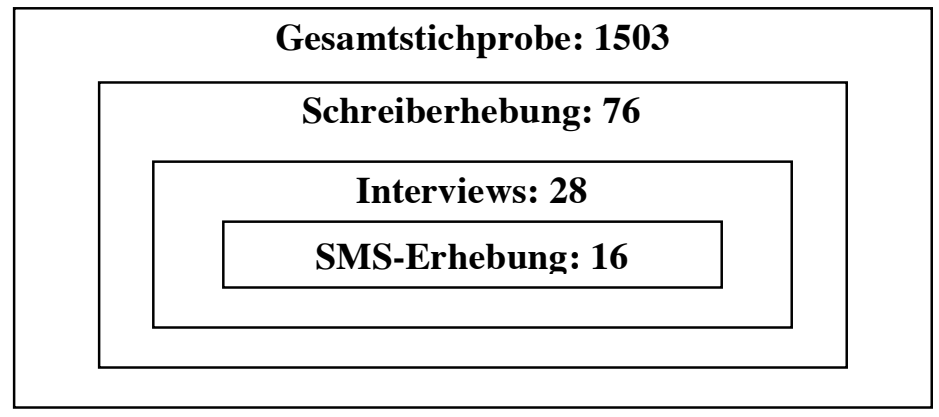

Abb. 1: Die Gesamtstichprobe und Teilstichproben des Projekts "Literale Resilienz"

\section{$3 \quad$ Konzepte des Lesens und Schreibens in der vorliegenden Studie}

Die Entwicklung der Kompetenzen, die im Zusammenhang mit Schriftlichkeit stehen, soll das Lesen und Schreiben gemeinsam in den Blick nehmen. Das ist ein zentrales Anliegen unseres Forschungsprojektes: Die Bedeutung der Interaktion von Lesen und Schreiben kann nicht nur aus den Anfängen des Lesenlernen ersehen werden - das mit der Methode des "Lesens durch Schreiben" gute Erfolge erzielt - sondern auch weit darüber hinaus. Lesen und Schreiben fördern sich gegenseitig in ihrer Entwicklung und ihrem Gebrauch. Lesen und Schreiben sind, historisch gesehen, verglichen mit dem alltäglichen Sprechen und Zuhören ausgeprägt elitäre Tätigkeiten. Von daher spielen auch heute noch elitär geprägte Konzepte von Schreiben und Lesen eine Rolle. Diese Konzepte sehen Lesen und Schreiben prototypisch als einsame, konzentrierte, länger dauernde, mit Planung und Korrekturen verbundene und als Arbeit empfundene Tätigkeit. Das "süchtige" eskapistische Lesen, das vor allem Leserinnen zugeschrieben wird, gilt zwar nicht als anstrengend, aber immerhin auch als "einsam" und "länger dauernd". Das entsprechende Pendant beim Schreiben, die Schreibsucht hingegen die u. a. den Zeitungsredaktionen in Form von Leserbriefen zugeht - ist seltener als das süchtige Lesen. Es wird eher von Männern praktiziert und mit Einmischung und (einsamem) Kämpfen assoziiert - nicht selten auf extremem oder verlorenem Posten.

Natürlich können sowohl Lesen als auch Schreiben solche einsamen, konzentrierten eher anstrengenden Tätigkeiten sein, aber das muss nicht sein. Für die breite Verankerung in der Bevölkerung ist wichtig, auch die alltäglichen Funktionen des Lesens und Schreibens wahrzunehmen und ihren Wert für den privaten und beruflichen Alltag zu erkennen und zu formulieren und damit auch zu valorisieren: Das Zeitungslesen am Küchentisch - eingebettet in bewertende Kommunikation der Küchengemeinschaft - oder das Schreiben von Notizen sind für die Ausbildung eines selbstverständlichen Gebrauchs der Lese- und Schreibkompetenzen ebenso wichtig wie das Lesen und Schreiben von Büchern, das nicht für alle Mitglieder der Gesellschaft eine Rolle spielt und spielen kann. Die Selbstverständlichkeit des Gebrauchs ist die Basis für die Entwicklung einer Situation der stärkeren sprachlichen Dekontextualisierung, die schwierigere Texte oft auszeichnet: Die Dekontextualisierung eines Textes bedeutet, dass er aus der Situation herausgelöst und dadurch in sprachlicher Hinsicht komplexer wird. Es gibt weniger Verweise auf die Situation und mehr Verweise innerhalb des Textes. Dafür werden sprachliche Mittel der Vertextung verwendet wie Pronomina, textstrukturierende Zeitformen, Verweise über mehrere Sätze hinweg und andere mehr (cf. dazu auch Häcki Buhofer 2007).

\footnotetext{
${ }^{1}$ Für einen ausführlicheren Projektbeschrieb cf. Schneider/Bertschi-Kaufmann (2006).
} 
Unsere Modellierungen des Lesens und Schreibens beziehen sich auf die gängigen Konzepte und modifizieren sie auch. Wir verwenden ein erweitertes "Kompetenzmodell Lesen", das die Lesetätigkeit nicht nur als Textverstehen, sondern auch als empathische Leistung, die Fähigkeit der Wahrnehmung von Motivationen fiktiver Personen und von Gefühlsbeziehungen beschreibt (siehe weiter unten).

Für das Schreiben gehen wir von einem Ansatz von funktionaler alltäglicher Schriftlichkeit aus (cf. Barton 1994, Häcki Buhofer 1983, 1989, 1995): Schreiben soll als Alltagshandlung in den Blick genommen werden wie Alltagssprechen auch. Es soll in seiner Funktion für die Schreibenden wie die Lesenden gesehen werden und dabei als ebenso spontan, ungeplant, sorglos und (weltgeschichtlich gesehen) belanglos wie das Alltagssprechen. Wir erteilen damit einem elitären Schreibkonzept eine Absage, weil es nicht der Realität der überwiegenden Mehrheit der Schreibenden entspricht, die nicht Schriftsteller sind oder sonst von Berufs wegen hauptsächlich schriftliche Texte verfassen, ohne uns von einem lehr- und lernbaren Konzept eines an Normen der Kommunikation orientierten Schreibens zu verabschieden, aber auch ohne die Rechtschreibung und kleine formale Fehler absolut dominant zu setzen.

\section{Aspekte der Forschungssituation im Bereich Hochspracherwerb von Kindern nicht-deutscher Erstsprache in der Deutschen Schweiz}

Es wird immer wieder gefragt, ob die Sprachsituation der Deutschschweiz, eine Diglossiesituation mit zwei funktional verteilten Varietäten, eine Schwierigkeit im Spracherwerb von Kindern nichtdeutscher Erstsprache darstelle. Bisherige Studien, welche sich dem Hochdeutscherwerb von mehrsprachigen Kindern und Jugendlichen in der Deutschschweizer Diglossiesituation gewidmet haben, konstatieren im besseren Fall spezifische Erwerbsbedingungen - die angesprochene Diglossiesituation - und spezifische lernersprachliche Phänomene. Im problematischeren Fall werden den Erwerbsbedingungen unbesehen erhöhte Anforderungen zugeschrieben und bei Mehrsprachigen allgemein geringere Kompetenzen vermutet. Dies geschieht ungeachtet der Dauer des Besuchs einer deutschsprachigen Schule und ungeachtet all der Faktoren, die - unter dem Konzept der Resilienz zusammengefasst - eine positive Schutzfunktion übernehmen können.

Ergeben sich also aus der Diglossiesituation besondere Anforderungen, Schwierigkeiten, Probleme für Deutsch als Zweitsprache Lernende? Oder ist eine Diglossiesituation ein weit verbreiteter Aspekt einer global gesehen "normalen" Sprachsituation, die sich im Verlauf des Spracherwerbs bewältigen lässt, ohne zu Auffälligkeiten zu führen? Wenn es um den frühen gesteuerten Fremdsprachenerwerb oder um bilingual aufwachsende Kinder geht, hat die Wissenschaft die kognitive Überbelastung, den "surcharge cognitive" (Lüdi 2007) zurückgewiesen. Sollte die durch die Lebenssituation gegebene Ausbildung von zweisprachigen Kompetenzen eine Hürde darstellen, die nicht alle Kinder nehmen können?

Die Diglossiesituation der Deutschschweiz hat ihre Besonderheiten (und ist dennoch kein Sonderfall). Weder hat die Schweizer Varietät kein Prestige (wie es für die Low-Variety gemäß klassischer Definition charakteristisch sein soll), noch trifft eine mediale Verteilung Hochdeutsch geschrieben, Schweizerdeutsch gesprochen - generell zu. Auch wird Hochdeutsch nicht nur gesteuert in der Schule erworben (cf. zu Konzept und Erwerbsprozessen Häcki Buhofer/Burger 1998).

Die bisher für die deutschsprachige Schweiz vor allem untersuchten Altersgruppen sind Kinder im Kindergarten und im frühen Schulalter. Der Hochdeutscherwerb von Kindern in der Deutschschweiz ist in den vergangenen 15 Jahren verschiedentlich Gegenstand größerer Projekte gewesen. Dabei hat sich gezeigt, dass die Kinder schon im Vorschulalter über z. T. erstaunliche passive, aber auch aktive Kenntnisse des Hochdeutschen verfügen und dass die 
Primarschule viel zu wenig mit diesem Vorwissen rechnet(e). Diese Kenntnisse werden "ungesteuert", also z. B. durch Medienkonsum oder Kontakt mit muttersprachlich hochdeutsch sprechenden Personen erworben. Die emotionale Distanz der DeutschschweizerInnen zum gesprochenen (nicht zum geschriebenen) Hochdeutschen wird gegenläufig zur ungesteuerten Entwicklung - während und in den ersten zwei Primarschulklassen durch allgemeine Sozialisationsprozesse und gegen die Intention der Lehrpersonen erworben (cf. Häcki Buhofer/Burger 1998). An diese Arbeiten knüpft die Studie von Karin Landert (2007) an. Sie untersucht die Problematik jedoch in einem speziellen und aktuellen Umfeld: im Zusammenhang mit dem QUIMS-Projekt ("Qualität in multikulturellen Schulen", d. h. in Schulen mit mindestens 50\% Anteil an Fremdsprachigen). In solchen Kindergärten und Schulen bietet sich Hochdeutsch als Umgangssprache auch bei jungen Kindern an. Es zeigt sich, dass die Kinder aus dem Kindergarten, in dem Hochdeutsch gesprochen wird, flüssiger, reichhaltiger und in längeren Texten sprechen. Allerdings ist ihr Hochdeutsch formal nicht besser. Lexikalisch sind Kinder der ersten Primarklasse durch ungesteuertes Lernen schon wesentlich weniger dialektnah als Kindergartenkinder. Interessant sind aber hier die Auffälligkeiten bei den mehrsprachigen Kindern in dieser frühen Phase der institutionellen Erziehung. Sie weisen im Gegensatz zu den muttersprachlich deutschen Kindern Verstöße gegen die Kongruenzregeln oder Auslassung von obligatorischen Artikeln sowie Kasusfehler auf. Sie verwenden auch besonders gern die periphrastische Konstruktion tun + Infinitiv. Ein erwerbstheoretisch interessanter Befund ist der, dass die Kinder mit fremdsprachigem Hintergrund stärker lexikalisch lernen, da sie nicht in gleicher Weise wie die muttersprachlich deutschen Kinder auf ein System von Transfer-Regeln zurückgreifen können.

Die Sprachbiographien einzelner Kinder, welche die Arbeit abrunden, zeigen wenigstens tendenziell, dass für die Kinder eine positive Einstellung der Eltern und der Lehrkräfte zum Hochdeutschen förderlich ist für den Erwerb. Dabei haben offensichtlich die fremdsprachigen Kinder einen problemloseren Zugang zum Hochdeutschen. Die individuellen Sprachprofile illustrieren des Weiteren, wie komplex die Spracherwerbsbedingungen besonders der mehrsprachigen Kinder sind und sie zeigen, dass der Einfluss der familiären, sozialen und medialen Umgebung gar nicht überschätzt werden kann. Die äußerst differenzierte Analyse ermöglicht erstmals eine vorsichtige Einschätzung der Wirksamkeit des Schulversuchs "Hochdeutsch auch im Kindergarten" aus linguistischer Perspektive.

Einen ganzen Komplex von Analysen und Forschungsberichten über den Hochdeutscherwerb von mehrsprachigen Kindern hat Mathilde Gyger erarbeitet, die u. a. einen vierjährigen Schulversuch "Standardsprache im Kindergarten" sprachwissenschaftlich begleitet hat, der 2005 abgeschlossen wurde. Dabei sollte die Frage beantwortet werden, ob und inwiefern sich eine Umstellung auf Standardsprache in Kindergärten mit einem hohen Anteil fremd- und mehrsprachiger Kinder (d. h. mehr als 75\%) empfiehlt. Aus dem Projekt "Standardsprache im Kindergarten" 2001-2005 sind folgende Resultate gewonnen worden: Die Ergebnisse zur mündlichen Kompetenz ergeben kein eindeutiges Bild hinsichtlich einer positiven Auswirkung der Standardsprache auf den Zweitspracherwerb an sich. "Die Sprachtests belegen vergleichbare Sprachfähigkeiten" bei mehrsprachigen Kindern in schweizerdeutschen bzw. hochdeutschen Kindergärten. "Die spontanen Sprachproben hingegen ergeben bei der Versuchsgruppe mit Standardsprache im Kindergarten eine stärkere Zunahme an Sprechfreude und Wortschatz als bei der Vergleichsgruppe mit Mundart im Kindergarten." (Gyger 2005: 8). Interessant ist die Schlussfolgerung, dass die Lehrkräfte Entscheidungshilfen bei der Verwendung von Vokabular aus dem Bereich der Grenzfälle des Standards brauchen und einen hochdeutschen Alltags-, Affekt- und Vulgärwortschatz benötigen: Von Finken bis zu Schnuder muss eine vertretbare Variante gewählt werden können. Ein weiteres Projekt 
"Deutsch-Standard im Kindergarten", das bis 2009 laufen soll, geht dieser Fragestellung weiter nach (Gyger 2006).

Wir schließen mit unserem Projekt an diese Arbeiten an, haben aber die Altersgruppe, die vor dem Abschluss der obligatorischen Schulzeit steht, in den Blick genommen. Außerdem haben wir nicht nur städtische, sondern auch ländliche Verhältnisse untersucht und haben es mit der ganzen Bandbreite von Schulklassen mit höheren und tieferen Anspruchsniveaus sowie mehr oder weniger fremdsprachigen Jugendlichen zu tun. Ein guter Teil der Jugendlichen wird die aktuellen Kompetenzen ins Erwachsenenleben mitnehmen und trotz Schulausbildung während der Lehre nicht mehr sehr viel Allgemeines dazulernen, jedoch lebenslang Wortschatz lernen und fach- und berufsspezifische Textsorten und Ausdrucksweisen erwerben.

Im Rahmen unseres erweiterten Konzepts von literalen Kompetenzen sind über Syntax und Wortschatz hinaus von Interesse:

- Reflexion von Vorteilen, Nachteilen der eigenen Sprachsituation, eigenen Kompetenzen, Konzepten von Schriftlichkeit, Mündlichkeit, Einstellung gegenüber den Varietäten, Wertschätzung, Nutzenüberzeugungen;

- Allägliches Schreiben und Lesen außerhalb der Schule von "SMS-Schreiben" bis "Game-Instruktionen-Lesen" oder "Fernseh-Lesen".

Die mehrsprachigen Kinder der Stichprobe verteilen sich wie folgt auf die verschiedenen Sprachgruppen:

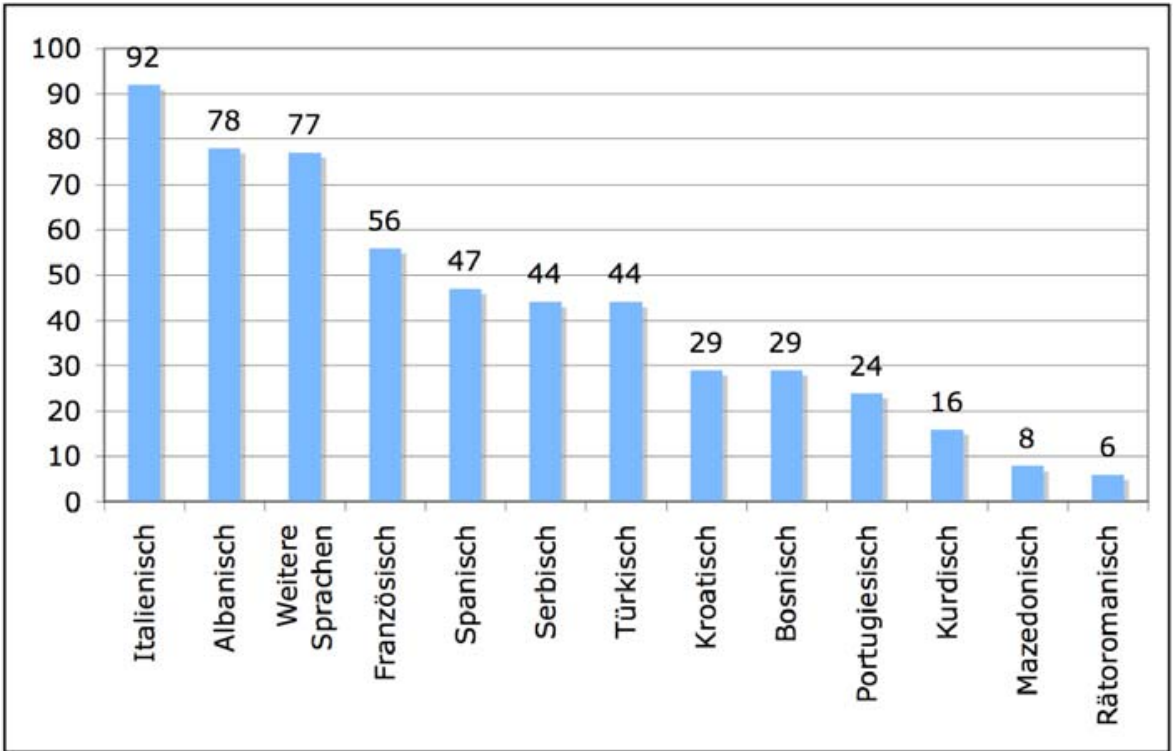

Abb. 2: Die Sprachgruppen in unserer Stichprobe 


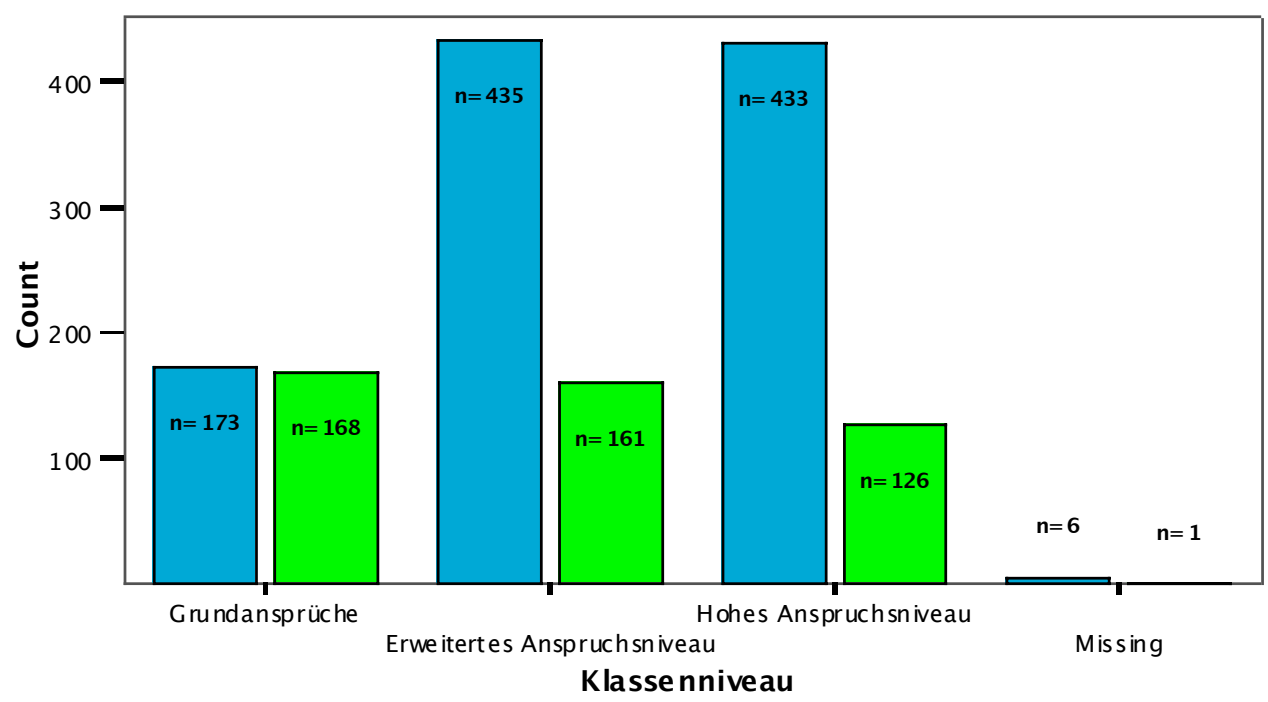

Meh rsprachigkeit

Deutschsprachig

$\square$ Mehrsprachig

Bars show counts

Abb. 3: Migration als Risiko - die ungleiche prozentuale Zuweisung von Ein- und Mehrsprachigen zu Anspruchsniveaus

Allgemein schulisch gesehen ist das mit der Migration verbundene Risiko auch in unserer Stichprobe offensichtlich: Während im Schultyp, der die Grundansprüche unterrichtet, nur 16\% einsprachige Schüler platziert sind, besuchen 36\% der Mehrsprachigen solche Klassen.

\section{Verwendung der Varietäten und diesbezügliche Einstellungen in der Diglossie- situation}

Die in diesem Kapitel wie in den beiden folgenden präsentierten Ergebnisse zu Kompetenzen, Einstellungen und Gewohnheiten bezüglich dem Schreiben und Sprechen in der Diglossiesituation stammen von mehrsprachigen Jugendlichen innerhalb der Teilstichproben der Schreiberhebung und der Interviews. Sechs davon besuchen seit 3-8 Jahren eine deutschsprachige Schule (nachfolgend "kurzansässige Mehrsprachige"), deren elf sind seit über 8 Jahren an einer deutschsprachigen Schule (nachfolgend "langansässige Mehrsprachige").

Bezüglich der literalen Aktivitäten in der Freizeit lässt sich eine situativ bedingte Verwendungsweise der Varietäten konstatieren; dabei zeigen sich keine Unterschiede zwischen Ein- und Mehrsprachigen: neben Hochdeutsch ist auch der Dialekt Lese- und Schreibsprache der meisten. ${ }^{2}$ Die Wahl der Varietät hängt zusammen mit einer Anpassung an die Varietät der KommunikationspartnerInnen und mit der jeweiligen Textsorte oder Textlänge. Die schriftliche Kommunikation mit KollegInnen in den neuen Medien (MSN, SMS) ist dialektdominiert; nicht-kommunikatives Schreiben wie beispielsweise das Verfassen von Geschichten demgegenüber findet überwiegend in der Hochsprache statt. Ansonsten bietet sich das Bild einer relativ heterogenen, indes personenbezogen stabilen Verwendungsweise: schriftliche Kommunikation mit Erwachsenen, Tagebuchschreiben und Briefeschreiben findet personenabhängig relativ konsequent auf Hochdeutsch oder Dialekt statt.

\footnotetext{
2 Die Beantwortung dieser Frage hängt vom Lesekonzept ab: möglicherweise haben diejenigen Vpn, welche den Dialekt nicht als private Lese- und Schreibsprache angegeben haben, die Lesetätigkeiten beim Chatten und SMSen nicht als "Lesen" betrachtet.
} 
Was das Sprechen anbelangt, weisen die Mehrsprachigen eine insgesamt häufigere Verwendung der Hochsprache auf, welche in gewissen Situationen an die Stelle des von Einsprachigen verwendeten Dialekts tritt. Bemerkenswerterweise wird die nachfolgend tabellarisch dargestellte Varietätenwahl auch so gehandhabt, wenn die KollegInnen aus dem Herkunftsland der befragten Jugendlichen stammen:

\begin{tabular}{|l|c|c|c|c|c|}
\hline SPRECHSPRACHE & Nur Dialekt & $\begin{array}{l}\text { Hochdeutsch } \\
\text { und Dialekt }\end{array}$ & $\begin{array}{l}\text { Hochdeutsch, } \\
\text { die Erstsprache } \\
\text { und Dialekt }\end{array}$ & $\begin{array}{l}\text { Die Erstsprache } \\
\text { und Dialekt }\end{array}$ & $\begin{array}{l}\text { Die Erstsprache, } \\
\text { aber } \\
\text { nicht Dialekt }\end{array}$ \\
\hline $\begin{array}{l}\text { Kurzansässige } \\
\text { Mehrsprachige } \\
\text { 6 Pers.) }\end{array}$ & 1 & 0 & 3 & 1 & 1 \\
\hline $\begin{array}{l}\text { Langansässige } \\
\text { Mehrsprachige } \\
\text { (11 Pers.) }\end{array}$ & 7 & 1 & 1 & 2 & 0 \\
\hline
\end{tabular}

Tab. 1: Sprachverwendung

Die Einstellungen gegenüber den Varietäten erlauben keine Unterscheidung von Ein- und Mehrsprachigen. So gilt der Dialekt als den eigenen Gedanken und Gefühlen näher, und man schreibt ihm eine persönlichere Wirkung auf die AdressatInnen zu; entweder wird er als einfacher betrachtet, da Groß- und Kleinschreibung sowie Orthographie nicht an feste Regeln gebunden sind, oder seine Verwendung für das private Lesen und Schreiben erfolgt aus purer Gewohnheit ohne Reflexion allfälliger kognitiver Erleichterungen. Für eine affektive Beziehung zum Hochdeutschen wiederum finden sich im Material weder für Ein- noch Mehrsprachige Belege. Allerdings wird das Lesen von längeren Texten auf Hochdeutsch bevorzugt, weil es als leichter empfunden wird; Gedrucktes (Zeitschriften, Bücher) lesen die Jugendlichen aus purer Gewohnheit auf Hochdeutsch, ohne dass dies weiter reflektiert würde. Einige Jugendliche empfinden die Regelgebundenheit der Hochsprache bei der Textproduktion gerade als Erleichterung, da diese ein schnelleres Schreiben erlaube, welches gerade für längere Texte erwünscht ist. Zudem schreiben die Jugendlichen der Hochsprache beim Schreiben bessere Verständlichkeit zu.

\section{6 (Alltägliche) Schriftlichkeit und Schreibkompetenzen als subjektive Konstrukte I}

\subsection{Die Bedeutung von Schreiben für die eigene Person sowie in der Gesellschaft und Schreibgewohnheiten}

Für Ein- und Mehrsprachige gilt, dass man insgesamt gerne schreibt. Die Vorstellungen von Schriftlichkeit sind durchaus alltäglich. Dies zeigt sich beispielsweise daran, dass auf die Frage nach der persönlichen Bedeutung von Schreiben die Sprache zunächst keineswegs auf das schulische Schreiben kommt, sondern vielmehr das Schreiben von Notizen, SMS, Chatbeiträgen, persönliches Schreiben in Form von Tagebuchführen, Geschichten- oder Gedichtschreiben oder das Verfassen von Briefen Erwähnung findet. Im Allgemeinen wird Schreiben als wichtig erachtet und geschätzt, wobei - altersbedingt - seine kommunikative Nutzung im Rahmen der Kontaktpflege im Vordergrund steht. Für die Mehrsprachigen ist hier besonders der schriftliche Austausch mit Kollegen im Herkunftsland hervorzuheben: die neuen Medien machen die Kontaktpflege billiger und schneller. Schreiben gilt überdies als unverzichtbare und praktische Technik, welche man beherrschen muss, will man in der Berufswelt und im gesellschaftlichen Alltag bestehen können. Es wird die Meinung vertreten, in allen Berufen müsse man lesen und schreiben. Wenn als Beispiele Schilderlesen auf der Baustelle, das Lesen von Anzeigetafeln oder Fahrplänen erwähnt werden, zeugt das erneut vom alltäglichen Verständnis von Schriftlichkeit. 


\subsection{Selbsteinschätzungen der Schreib- und Sprechkompetenzen}

Die quantitative Befragung ergibt, dass bezüglich der Selbsteinschätzung der drei erfragten Schreibkompetenzen "Abstraktion/Komplexität", "Orientierung am Ich" und "Normorientierung"3 keine Unterschiede zwischen Ein- und Mehrsprachigen nachweisbar sind. Bei der "Diglossiefrage" "Ich kann Hochdeutsch schreiben, ohne dass Mundart darin vorkommt" erreicht das Sample der Mehrsprachigen $(\mathrm{n}=461)$ auf einer Skala von 1 ("stimmt gar nicht") bis 4 ("stimmt völlig") einen Wert von 3.24; die Einsprachigen $(\mathrm{n}=1027)$ erreichten bei derselben Frage den unwesentlich höheren Wert von 3.31. Leichte Differenzen ergeben sich bei der Selbsteinschätzung der Jugendlichen bezüglich ihrer Sprechkompetenzen im Dialekt: die Dialektkompetenz beim Sprechen wird bei den kurz- und langansässigen Mehrsprachigen in den meisten Fällen als "sehr gut", einige Male als "gut" eingeschätzt.

Insgesamt lässt sich für die von uns untersuchte Stichprobe von langansässigen Mehrsprachigen nicht mehr nachweisen, dass die Diglossiesituation in der subjektiven Wahrnehmung besondere Anforderungen oder Schwierigkeiten mit sich bringt.

\subsection{Schreibkompetenzen "objektiv bestimmt": funktional-alltägliche Betrachtungs- weise}

Alltägliches Schreiben erfordert keine maximale Strukturiertheit, Explizitheit oder Einhaltung von Schreibkonventionen, muss aber den jeweiligen Kommunikationsbedingungen angemessen sein. Ein grammatisch und orthographisch korrekter Text braucht noch nicht angemessen zu sein, wohingegen ein in Bezug auf die höheren Schreibkompetenzen der sozialen und funktionalen Kohärenzherstellung guter Text durchaus grammatische und orthographische Mängel aufweisen kann. ${ }^{4}$ Wir richten den Fokus auf die Bedingungen von Schreibhandlungen und deren Berücksichtigung in der Vertextung, also die auf vielfältige Art und Weise $\mathrm{zu}$ erreichende, soziokulturell bestimmte Situations-, Adressaten- und Zweckadäquatheit von Texten. Den Grad der Adäquatheit haben wir anhand eines funktionalpragmatischen Aufgabenprofils und entsprechenden pragmatischer Teilkompetenzen zu ermitteln versucht.

\subsection{1 "Objektive Bestimmung" der Schreibkompetenzen}

Zur Ermittlung der funktional-alltäglichen Schreibkompetenzen wurden einer Stichprobe von 76 Jugendlichen, darunter 19 mehrsprachigen, zwei Schreibaufgaben vorgelegt, ein Argumentationsbrief sowie eine Überarbeitungsaufgabe. ${ }^{5}$ Die Aufgabenstellungen waren so angelegt, dass die Kompetenz erforderlich war, einen Text den Bedingungen der medialen Situation angepasst und unter Einhaltung der soziokulturell gültigen Interaktionsnormen ziel-

\footnotetext{
${ }^{3}$ Die Selbsteinschätzung der Schreibkompetenz wurde mit drei Faktoren erfasst: der "Normorientierung", bei welcher die Kompetenz, normgemäss zu schreiben, im Vordergrund steht (z. B. "Ich kann ohne Rechtschreibfehler schreiben"); der "Orientierung am Ich", welche auf die Kompetenz zielt, eigene Gedanken oder Meinungen zu versprachlichen (z. B. "Ich kann gut über meine eigenen Erfahrungen schreiben"); der "Abstraktion/Komplexität", mit welcher die Kompetenz angesprochen wird, über Abstraktes und Komplexes schreiben zu können (z. B. "Ich kann in Aufsätzen Argumente und Gegenargumente gegeneinander abwägen"). Die Faktoren wurden im Rahmen der Studie von Kassis/Schneider (2004) entwickelt und konnten für das Resilienzprojekt übernommen werden.

${ }^{4}$ Von "höheren Schreibkompetenzen" sprechen wir deshalb, weil sie implizieren, dass die Schreibenden den Text als ganzen, in seiner Funktion innerhalb einer bestimmten kommunikativen Situation bewusst oder unbewusst erfassen können. "Weniger voraussetzungsreiche Schreibkompetenzen" demgegenüber erfordern "nur" die Erfassung des Kontextes des jeweiligen Wortes oder Satzes.

${ }^{5}$ In der Überarbeitungsaufgabe waren die Jugendlichen aufgefordert, einen auf verschiedenen Ebenen des Textes mangelhaft formulierten Brief adressaten- und zielorientiert zu verbessern; es kann hier nicht näher darauf eingegangen werden.
} 
und adressatenorientiert zu verfassen bzw. zu überarbeiten. Im Argumentationsbrief wurde die folgende Situation vorgegeben: ${ }^{6}$ die Jugendlichen waren nach einem Badeausflug mit Freunden mit 2. Klasse-Billetten aus Versehen in der 1. Klasse gefahren, da sie unter Zeitnot den Zug unmittelbar vor Abfahrt erreichten und aufgrund der Ähnlichkeit der Sitzbezüge in beiden Klassen deren Unterscheidung übersahen. ${ }^{7}$ Dabei gerieten sie in eine Kontrolle, in welcher ihnen Bussen von 80 Franken erteilt wurden. Diese Behandlung empfanden sie als höchst ungerecht und beschlossen, dem Chef des Zentralkundendienstes der SBB, einem fiktiven Herrn Bernegg, einen Beschwerdebrief zu schreiben mit der Bitte um Bußenerlass.

Um die Jugendlichen von der Inhaltsgenerierung zu entlasten und damit die Kompetenzen zur Herstellung eines inhaltlich und sozial kohärenten Textes zu isolieren, wurde den Jugendlichen eine Auswahl von 9 unterschiedlich angemessenen Argumenten vorgegeben, aus welchen sie drei auszuwählen und in ihren Text einzugliedern hatten. Die Aufgabe verlangte explizit und in der nachfolgend genannten Reihenfolge, den Chef des Zentralkundendienstes einerseits über den Ereignishergang $\mathrm{zu}$ informieren und ihn andererseits um den Erlass der Buße zu bitten. Zur Lösung stand die Zeit von 30 Minuten zur Verfügung.

Beispieltext einer Mehrsprachigen:

Sehr geehrter Herr Bernegg

Ich möchte sie nochmals bitten die Busse zurück zu ziehen.

Ich finde es sehr ungerecht das was passiert ist.

Es verlief nählich so: Ich und meine Freunde waren am See und als wir zurück zum Bahnhof liefen trafen wir noch eine Kollegin und redeten noch ein bisschen. die Zeit wurde sehr knapp, also verabschiedeten wir uns von ihr und gingen schnell die Billette lösen. Wir lösten sie für die 2. Klasse. Wir mussten rennen sonst verpassten wir noch den Zug also stiegen wir so schnell wie möglich in den Zug, wir schauten nicht ob wir jetzt in der 1. Klasse waren oder in der 2. Klasse, weil wir rennen mussten und keine Zeit mehr da war um zu schauen in welcher Klasse wir jetzt waren. Ich finde es ungerecht, dass Leute, die gar keine Fahrkarte kaufen und offensichtlich schummeln. gleich behandelt werden sollen wie solche, die eine gekauft haben, sich dann aber ohne es zu merken in die falsche Klasse gesetzt haben.

Wir würden es Ihnen sehr danken, wenn Sie ein wenig Nachsicht zeigen könnten und die Busse zurückziehen würden.

mit freundlichen Grüssen

Beispieltext eines Einsprachigen:

Sehr geehrter Herr Bernegg

Leider sind ich und meine beiden Kollegen Opfer eines dummen Zufalls geworden. Wir haben uns nämlich nicht absichtlich in das 1 . Klass Zugabteil gesetzt. Wir hatten unsere restliche Zeit falsch eingeschätzt um zum Bahnhof zu gelangen. Nachdem wir unsere Billets gelöst hatten, stiegen wir kurzerhand in den erst besten Wagong ein, ohne darauf zu achten, ob es nun 1. oder 1. Klasse ist. Bevor wir uns im Klaren waren, wo wir nun waren, kam einer Ihrer Billetkontrolleure geradewegs auf uns zu. Ohne das wir unser Argumente im Bezug

\footnotetext{
${ }^{6}$ Um möglichst weitgehend zu vermeiden, dass die Jugendlichen beim Schreiben größere Textteile von der Aufgabenstellung übernehmen konnten, erfolgte die Situationsdarstellung in der Form eines schriftlich festgehaltenen Gesprächs, in welchem die einzelnen Beiträge wie in einem Interview mit den Namen der Sprechenden eingeführt wurden und so weit als möglich auf Situationsbeschreibungen verzichtet wurde.

${ }^{7}$ Eine im Kanton Zürich tatsächlich vorliegende Situation: die erste und zweite Klasse in S-Bahn-Zügen unterscheiden sich allein durch die weißen Kopfteilbezüge in der ersten Klasse.
} 
des Abteils vorbringen konnten, beschuldigte der Kontrolleur uns, uns absichtlich in die erste Klasse eingeschmuggelt zu haben. Trotz unseren Bemühungen bekamen wir einen Bussezettel, den wir unterschreiben mussten. Ich hoffe Sie sehen jetzt ein, warum wir mit dieser Entscheidung nicht zufrieden sind. Und hätte wir schummeln wollen, hätten wir uns doch gar nicht erst die Mühe gemacht Billete zu kaufen und Ihnen diesen Entschuldigungsbrief zu schreiben.

Darum bitte ich Sie die Busse zu erlassen. Ich werde mich in Zukunft darauf konzentrieren meine Defizite im "Zug einsteigen" zu verbessern.

Ich danke Ihnen vielmals und entschuldige mich auch im Namen meiner Freunde.

Mit freundlichen Grüssen

\subsubsection{Funktionale Bewertung der Schreibkompetenzen}

Die Texte wurden anhand eines funktional-pragmatischen Aufgabenprofil bezüglich sieben zu lösender Teilaufgaben nach einer dreistufigen Niveauskala geratet, wobei zwischen guten, mittelmässigen und schlechten Realisierungen unterschieden wurde. ${ }^{8}$ Eine erste pragmatische Teilkompetenz bezog sich auf die Herstellung eines Textes im engeren Sinne, welche mittels der folgenden Aspekte operationalisiert wurde: Textordnung, Textklammer, Kontextualisierung des Sachverhalts. Eine zweite pragmatische Kompetenz bezog sich auf diejenigen Aspekte des Textes, welche interaktive Kompetenzen voraussetzen wie: Realisierung des Appells, Stützung der Bitte/Strittigkeit, Verwendung von sprachlichen Höflichkeitsmitteln, Einbezug der Adressatenperspektive. ${ }^{9}$ Die Einhaltung konventioneller Schreibnormen wie die Regeln von Orthographie und Interpunktion wurden in der Bewertung nicht berïcksichtigt.

\footnotetext{
${ }^{8}$ Alle 78 Texte wurden von 4 Raterinnen isoliert beurteilt; nichtübereinstimmende Urteile wurden in der Gruppe diskutiert; nur wenn diskursiv eine Übereinstimmung erzielt werden konnte, galten die Beurteilungen bezüglich dieser Punkte ebenfalls als Übereinstimmungen. Interrater-Übereinstimmung:

Bei 103 von 133 Beurteilungen: 100\% Übereinstimmung

Bei 24 von 133 Beurteilungen: 3 von 4 gleiche Beurteilung

Bei 6 von 133 Beurteilungen: keine Übereinstimmung.

${ }^{9}$ Bei den einzelnen Kriterien wurden die folgenden Texteigenschaften bewertet:

Textordnung: ist eine globale oder lokale Textordnung erkennbar, d. h. liest sich der Text ohne oder mit Brüchen?

Textklammer: sind Einleitung und/oder Schluss (allenfalls in Ansätzen) realisiert?

Kontextualisierung des Sachverhalts: wird der Sachverhalt mit den für die Verständlichkeit notwendigen "Welt"Ausschnitten dargestellt, verliert sich der Text in überflüssigen Details oder ist er zu knapp und bietet somit ein angesichts der kommunikativen Absicht unzureichendes Bild der Situation?

Realisierung des Appells: ist die Aufforderung bzw. Bitte an den Adressaten erwachsen-vernünftig und höflich versprachlicht, wird sie kindlich-flehentlich oder unhöflich formuliert?

Stützung der Bitte/Strittigkeit: werden Argumente vorgebracht, die bei einem Erwachsener in unserer Sprachgemeinschaft und in der Situation eines Herrn Bernegg Aussicht auf Erfolg haben, sind sie kindlichtreuherzig, (zu) emotional oder nicht-relevant?

Verwendung von sprachlichen Höflichkeitsmitteln: wird die Strittigkeit (die als ungerecht empfundene, aber aufgrund der gültigen gesetzlichen Regelung erfolgte Busse) mit abschwächenden, Debattierraum schaffenden, Verhandlungsbereitschaft signalisierenden Mitteln, direkt bzw. gar unhöflich vorgebracht?

Einbezug der Adressatenperspektive: werden die Interessen, die Situationswahrnehmung, Gegenargumente und allfällige zukünftige Handlungen des Adressaten berücksichtigt?
} 


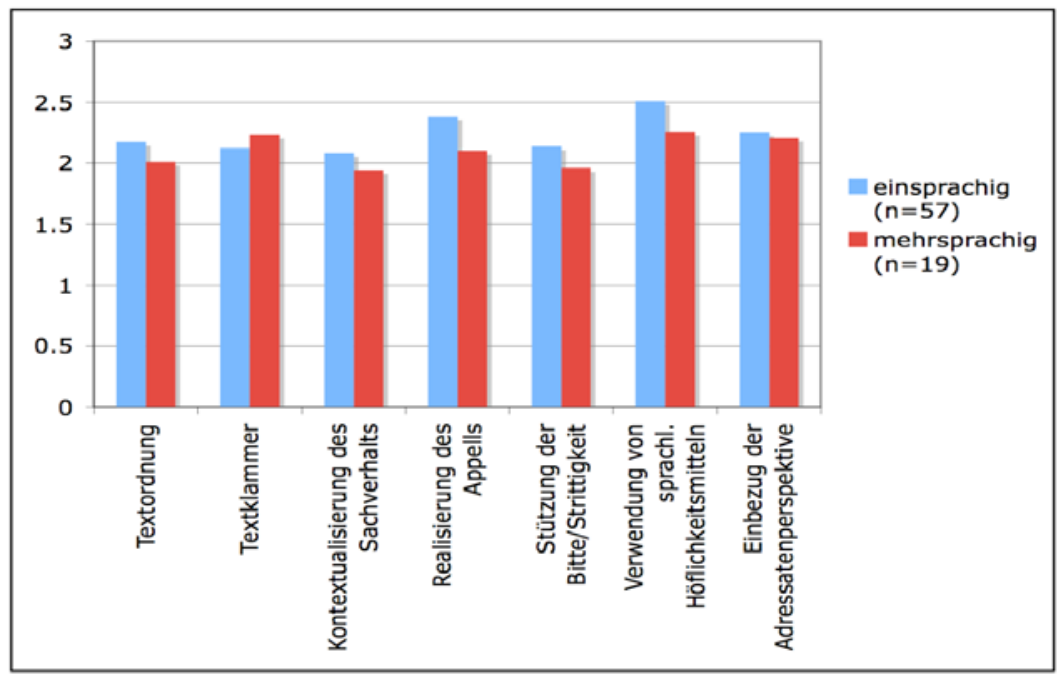

Abb. 4: Funktionale Bewertung der Schreibkompetenzen

\subsubsection{Pragmatische Schreibkompetenzen im Vergleich}

Die ermittelten Schreibkompetenzen ergeben folgendes Bild: mit wenigen Ausnahmen haben die ein- wie mehrsprachigen Jugendlichen die Anforderungen der Aufgabenstellung mittelmäßig bis gut bewältigt; die Texte der Mehrsprachigen sind bei allen sieben Bewertungspunkten minim schlechter als diejenigen der Einsprachigen. Bei der vorliegenden Stichprobengröße kann von keiner Signifikanz gesprochen werden. Dialektale Elemente sind kaum vorhanden; vereinzelt treten sie in der Lexik oder bei Phraseologismen auf. Konzeptionell mündliche Elemente demgegenüber sind sowohl bei Ein- als auch bei Mehrsprachigen häufig anzutreffen; betroffen sind die Ebenen Text, Syntax und Lexik sowie die pragmatische Dimension. Als auffälliges Phänomen innerhalb letzterer können eine mündliche Gesprächssituation simulierende Elemente wie Fragen oder die Nennung des Namens des Adressaten in der Briefmitte angeführt werden. Ein- wie mehrsprachige Jugendliche bedienen sich pragmatischer Formeln, insbesondere in der Ausgestaltung von Briefschlüssen. Topoi werden gehäuft in argumentativen Textteilen zur Stützung der Aufforderung eingesetzt; Sprichwörter werden nur in Einzelfällen verwendet. ${ }^{10}$

\section{7 (Alltägliche) Schriftlichkeit und Schreibkompetenzen als subjektive Konstrukte II}

\subsection{Textwissen und Textkompetenzen}

Auffällig ist, dass sich in der Fallpaar-Stichprobe $(n=16)$ im Gegensatz zur Stichprobe der Schreiberhebung $(n=78)$ bei den Mehrsprachigen keine wirklich guten Texte finden, sondern mittelmäßige und schlechte, wohingegen die Texte der Einsprachigen innerhalb der FallpaarStichprobe größtenteils als gut bewertet worden sind. Gleichzeitig ist bei den Interviews festgestellt worden, dass die Einsprachigen einerseits im Gesamt komplexere Vorstellungen von Textualität zum Ausdruck bringen als die Mehrsprachigen und über eine größere Bandbreite an Beurteilungskriterien für eigene und fremde Texte verfügen; andererseits nennen die Einsprachigen individuell eine höhere Zahl von Aspekten aus dieser Bandbreite und erwähnen als erstes pragmatische und semantische Aspekte und erst an zweiter Stelle

\footnotetext{
${ }^{10}$ Es wird eine genaue Analyse der eingesetzten sprachlichen Mittel vorgenommen. An dieser Stelle kann keine ausführliche Diskussion derselben erfolgen; stattdessen wurden diejenigen Mittel und Phänomene herausgegriffen, welche im Kontext der Fragestellung interessant sind, z. B. weil sie wie der Einsatz von pragmatischen Formeln auf eine gewisse Routine und damit Vertrautheit mit dem Verfassen von schriftlichen Texten - notabene auf Hochdeutsch - hindeuten.
} 
Annelies Häcki Buhofer/Hansjakob Schneider/Christine Beckert: Mehrsprachige Jugendliche im 61 Umgang mit Dialekt und Hochsprache in der Deutschen Schweiz

normbezogene. Der Kontext der Fragestellung waren dabei Schwierigkeiten beim Schreiben und diesbezügliche Bemühungen sowie Aussagen zu Schreibvorbildern: ${ }^{11}$

Die von Einsprachigen genannten Aspekte

- Pragmatik, Semantik und den Text als Ganzen betreffend: die Wirkung auf die LeserInnen, Konzentration auf das Thema/das Wesentliche, Inhaltsgenerierung (Ideen fehlen), Verständlichkeit, gute Wortwahl, Schreibstil/Tonlage, schwierige Textgattungen im privaten und schulischen Bereich, inhaltliche Qualitäten (interessant schreiben), Gliederung/Aufbau mit vorheriger Planung, Abwechslung/keine Wiederholungen.

- $\quad$ Die Einhaltung von Konventionen betreffend: Orthographie, Interpunktion, Grammatik.

Die von Mehrsprachigen genannten Aspekte

- Pragmatik, Semantik und den Text als Ganzen betreffend: Inhaltsgenerierung (Ideen fehlen), Konzentration auf das Thema/das Wesentliche, Verständlichkeit, keine Wiederholungen, Finden des treffenden/passenden Wortes, Textaufbau, Lücken im Wortschatz, Spannung erzeugen.

- $\quad$ Die Einhaltung von Konventionen betreffend: Orthographie, korrekte Formulierungen, Satzstellung, Groß-/Kleinschreibung.

Die Auffälligkeit des gemeinsamen Auftretens des Grades der Komplexität der Textvorstellung und desjenigen der Qualität der Textproduktion sei nachfolgend an den Vorstellungen von Textualität der Mehrsprachigen und des Einsprachigen, deren Texte weiter oben vorgestellt wurden, illustriert ${ }^{12}$ :

Aussagen zu Textualität der Mehrsprachigen:

I: Gitts Sache, wo du am Schriibe speziell schwirig findsch?

A: Wenni eifach so Wörter nid weiss, wie mer sie schriibt, aber schüsch... (...) Nei, eigendlich niid. Ebe eifach nur, weni so Wörter nid weiss.

I: Ähm känsch du jetz Lüüt, wo irgendwie speziell guet chönd schriibe?

A: Ja, mini Brüedere. (...) weni sie aube gseh und für es Diktat oder so lehre, gsehni eifach aube so so Wörtli, woni gar nid kenne und wo sie so guet chönne schriibe, und amix jo froogi sie ou, was s überhoupt heisst und so.

\section{Aussagen zu Textualität des Einsprachigen:}

A: Äs isch äso, i mache eifach dert, i mache eifach ds Thema, wo me müesst bim ne Erörterigsufsatz. Aber när chunnt mer ging no so viu drum ume Züg dri ine. Dass äs eifach aus när viu z viu isch.

I: Und wenn du seisch, dass dini Kollegin super cha schribe, aso was isch denn so super dra? Aso was gfallt dir so speziell guet?

A: Aso dr Inhaut u au das. Aso me chunnt eifach/s isch churz und bündig u me chunnt drus. U was me/ aso was sie wott $u$ au das.

Der Text der Mehrsprachigen ist bezüglich aller Bewertungskriterien mit Ausnahme der Realisierung des Appells als mittelmäßig eingestuft worden (der Appell als gut); der Text könnte zwar in dieser Form abgeschickt werden und würde vielleicht sein Ziel sogar

\footnotetext{
${ }^{11}$ Die Reihenfolge der Aufzählung folgt nicht der Häufigkeit der Nennung, sondern stellt im Sinne einer Darstellung der Vielfalt die Bandbreite der Aspekte von Textwissen dar; kursiv gesetzt sind Vorstellungen, die sich entweder nur bei Ein- oder nur bei Mehrsprachigen finden.

12 "I" steht für Interview-Frage; "A" steht für "Antwort der Jugendlichen".
} 
erreichen, indes wirkt er durch Wiederholungen sowie die Wahl eines umgangssprachlichen Registers stellenweise "hölzern", und der Text weist eine strenge Orientierung sowohl an der Aufgabenstellung wie auch an Textkonventionen auf. Bezüglich aller Kriterien als für gut befunden worden ist demgegenüber der Text des Einsprachigen; er lässt das Bemühen um eine originelle Wortwahl erkennen und inszeniert eine Beziehung zwischen unfreiwillig Schuldigen und einem wohlwollenden Herrn Bernegg, der die Situationsschilderung mit einem nachsichtigen Schmunzeln liest und wohl der Bitte der Besserungsbeflissenen nachgibt. Der Analyse der Produktionshaltung fügt sich das geäußerte Textwissen passend an: scheint für die mehrsprachige Jugendliche Schreibenkönnen äquivalent zu sein mit dem Beherrschen von Normen und dem Verfügen über einen großen Wortschatz, rückt der Einsprachige wirkungsbezogene und inhaltliche Aspekte in den Vordergrund.

Angesichts dieses Befundes wird die Hypothese aufgestellt, dass einerseits eine komplexere Vorstellung von Textualität die Adressaten- und Zielorientierung positiv beeinflussen kann, dass andererseits die Ausbildung einer komplexeren Vorstellung von Textualität bei Mehrsprachigen durch andere Faktoren behindert werden kann als bei Einsprachigen. ${ }^{13}$ Zur Begründung dieser erst anhand eines kleinen Samples $(n=16)$ aufgestellten Hypothese bieten sich verschiedene Ansätze. Zum einen könnte man von einem cognitive overload ausgehen: dass die Hürde des Zweitspracherwerbs für Einige doch $\mathrm{zu}$ hoch ist und sie in einer Semilingualität stecken bleiben. Eine andere Erklärung setzt beim Unwillen an, Schwächen einzugestehen (cf. Kontext der Interviewfrage): dass die mehrsprachigen Jugendlichen sich als kompetente Zweitsprachnutzende darstellen wollen. Diese Motivation könnte sich dann entweder darin äußern, dass sie sich auf messbare/harte Kriterien wie Rechtschreibung konzentrieren, oder überhaupt finden, sie hätten beim Schreiben keine Schwierigkeiten. Drittens könnte man sich denken, dass die Mehrsprachigen in einzelnen Fällen leicht im Hintertreffen sind bezüglich den "Feinheiten der Sprache". Hier wäre denkbar, dass ein bei ausgedehnter Lesepraxis in der Erstsprache entwickeltes Gespür für stilistische Feinheiten sich positiv auf die Zweitsprache auswirken könnte - gerade diese aber wurde bei den in der Fallpaar-Stichprobe untersuchten mehrsprachigen Jugendlichen kaum gepflegt. Des Weiteren darf man nicht ausschließen, dass es sich bei den in unserem soziokulturellen Verständnis relativen Mängeln um uns nicht vertraute kulturspezifische Aspekte von Schreiben handeln kann. Schließlich ist denkbar, dass die Lehrpersonen mehrsprachige Jugendliche vermehrt auf messbare Schreibkompetenzen hin schulen, im Bestreben, diejenigen Textaspekte, welche gemäß traditioneller Auffassung zu gutem Schreiben gehören, zu fördern.

\subsection{Fazit}

In Abhängigkeit von der angesetzten Schreibnorm fällt die Beurteilung der Schreibkompetenzen unterschiedlich aus. In Bezug auf grammatikalische und orthographische Aspekte konnten zumindest in fortgeschrittenem Lernalter keine Defizite der Mehrsprachigen, welche Deutsch als Zweitsprache in einer Diglossiesituation lernen, gegenüber den Einsprachigen festgestellt werden, während Grammatik und Orthographie wohl in den ersten Lernjahren eine Schwierigkeit für Mehrsprachige darstellen können. Die funktionalpragmatischen Schreibkompetenzen ihrerseits erlauben bereits bei früherem Lernalter und erst recht später keine Unterscheidung von ein- und mehrsprachigen SchreiberInnen.

\footnotetext{
${ }^{13}$ Es sei bemerkt, dass beide Jugendlichen die Sekundarschule (das mittlere Niveau der Oberstufe) besuchen; man kann also grundsätzlich von ähnlichen Erwartungen an die Textproduktion der SchülerInnen ausgehen; indes ist es ein Gemeinplatz zu betonen, dass die Unterrichtsinhalte lehrpersonenabhängig stark schwanken was den beiden Lehrpersonen der Jugendlichen beim schulischen Schreibunterricht wichtig war, wurde bei diesem Interview nicht erhoben.
} 
Ungeachtet dessen und wie der konkrete Fallvergleich zweier Vorstellungen von Textualität und der entsprechenden Schreibleistung gezeigt hat, ist es der Ausbildung umfassender funktional-pragmatischer Schreibkompetenzen von Mehrsprachigen - wie im Übrigen derjenigen vieler Einsprachiger auch - förderlich, wenn das Bewusstsein für die verschiedenen Ebenen von Sprache bzw. von Texten geschärft wird. Dies sollte beinhalten, dass Texte im Unterricht nicht auf Mittel zur Darstellung von Welt im Rahmen gültiger Normen und Regeln reduziert werden, sondern dass Strategien vermittelt werden, wie man beim Schreiben eine kommunikative Situation unter Einschluss der Beziehung zwischen Schreibenden und Adressierten, der Identitäten, der Meinungen und Interessen gestalten und damit gewissermaßen neu schaffen kann. Dem kreativen Moment von Sprachgebrauch, welches sich dennoch innerhalb soziokulturell akzeptablen Grenzen bewegt, kommt in diesem Zusammenhang große Bedeutung zu. Hierbei kann durchaus an die private Schreibpraxis angeschlossen werden, stellt diese sich doch gerade auch bei den Mehrsprachigen mit dem Verfassen von Tagebüchern, Gedichten oder Geschichten als vielfältig dar. Auch sollte das Bewusstsein für die verschiedenen Dimensionen eines Kommunikationsereignisses geschärft werden: als Ausdruck von eigenem Erleben, in der Ausrichtung auf AdressatInnen hin, als Darstellung der Sache und von der Gestaltung des Textes her. ${ }^{14}$ Bedeutsam ist auch die Würdigung der Erstsprache und die Anknüpfung an den sprachlichen Kompetenzen in dieser, wie es von verschiedenen Studien bereits empfohlen worden ist. ${ }^{15}$

Zeigen sich also zu einem späteren Zeitpunkt im Zweitspracherwerb in der Diglossiesituation keine nennenswerten Schwächen bei den Einstellungen und Kompetenzen der von uns untersuchten mehrsprachigen Jugendlichen mehr, würden die ebengenannten Massnahmen dazu beitragen, ausreichende funktional-alltägliche Schreibkompetenzen weiter $\mathrm{zu}$ entwickeln.

\section{$8 \quad$ Jugendliche und ihre Lesekompetenzen}

\subsection{Grundlegendes zur Lesekompetenz}

Die bisherigen Ausführungen haben eines deutlich gezeigt: Was die höheren, konzeptuellen und pragmatischen Schreibkompetenzen betrifft, so sind zwischen den fünfzehnjährigen Einsprachigen und Mehrsprachigen unserer Stichprobe keine signifikanten Unterschiede festzustellen. In diesem Fazit zeigt sich, dass es von Bedeutung ist, welche Teilkompetenzen des Schreibens fokussiert werden und an welchen Normen man Texte messen soll.

Die Frage nach dem Kriterium für Kompetenz stellt sich für das Leseverstehen in ähnlicher Weise. Breiter abgestützt als im Falle des Schreibens, weisen beim Lesen die Resultate großflächiger Untersuchungen, nämlich der PISA-Studien, nachdrücklich darauf hin, dass in der Schweiz Mehrsprachige weniger lesekompetent sind als Einsprachige (Moser 2002). Wir dürfen für unsere Stichprobe also von der Hypothese ausgehen, dass Mehrsprachige in den von uns eingesetzten Tests schwächere Resultate erbringen als Einsprachige. Allerdings dürfte die Deutschschweizer Diglossiesituation im Falle des Leseverstehens kaum als direkter Prädiktor für die Schwierigkeiten der Mehrsprachigen anzusehen sein. Verstehensprozesse, wie sie Häcki Buhofer et al. (1994) und Schneider (1994) für den frühen und ungesteuerten Erwerb des Standarddeutschen durch Deutschschweizer Kinder beschreiben, sind im Alter von fünfzehn Jahren und bei mehrheitlich fortgeschrittenem Stadium des Standarddeutsch-

\footnotetext{
${ }^{14}$ Cf. die Empfehlungen in Gyger/Heckendorn-Heinimann (1998: 129): "Für den Schulerfolg muss die gesamte Sprachkompetenz ausschlaggebend sein; die Beurteilung darf nicht nur auf einzelnen Spezialfertigkeiten (Rechtschreibung, Fall-Endungen) basieren".

15 Cf. beispielsweise Luchtenberg (2002), Gyger /Heckendorn-Heinimann (1998).
} 
erwerbs nicht mehr zu erwarten. Hingegen mögen wenig umfangreiche Lesepraxis und Beschränkungen des Wortschatzes zu Schwierigkeiten führen.

Im Anschluss an die Veröffentlichung der Resultate der PISA-Studie 2000 zum Leseverstehen hat sich in fachdidaktischen Kreisen eine rege Diskussion darüber entwickelt, ob das Lesekompetenz-Konzept von PISA für den schulischen Unterricht Weg leitend sein könne. Lesekompetenz wird aus der Perspektive von PISA primär als ein wichtiges Mittel verstanden für das Erreichen persönlicher Ziele, als Bedingung für die Weiterentwicklung des eigenen Wissens und der eigenen Kompetenzen und als Voraussetzung für die Teilhabe am gesellschaftlichen Leben (Artelt et al. 2004: 141). In dieser pragmatischen Tradition stellt ,Reading Literacy' eine Basisqualifikation dar, die in der modernen Gesellschaft für eine in beruflicher und gesellschaftlicher Hinsicht erfolgreiche Lebensführung unerlässlich ist (Hurrelmann 2007: 21). Von diesen Anforderungen her bestimmt sich, welche Texttypen und welche Aspekte des Lesens für die Messung der "Reading Literacy" einbezogen werden (cf. Bertschi-Kaufmann/Schneider 2006): Textseitig sind dies neben wenigen Erzählungen insbesondere Sach- und Informationstexte, mehrheitlich in fortlaufend geschriebenen Formaten (kontinuierliche Texte), teils aber auch mit bildhaften Darstellungen wie Diagramme, Bilder, Karten, Tabellen oder Graphiken (nichtkontinuierliche Texte).

Aufgabenseitig unterscheidet PISA drei Aspekte des Lesens, die quer zu den verschiedenen Texttypen berücksichtigt werden:

- Informationen ermitteln

- $\quad$ Ein allgemeines Verständnis des Textes entwickeln; eine textbezogene Interpretation entwickeln

- $\quad$ Über den Inhalt des Textes reflektieren; über die Form des Textes reflektieren (Artelt et al. 2004: 143)

Anders verhält es sich mit dem Kompetenzbegriff, welchen die deutsche Lesesozialisationsforschung entwickelt hat. Hier ist die Leitidee nicht das Lesen als rationale Selbstbestimmung, sondern das "gesellschaftlich handlungsfähige Subjekt" (Hurrelmann 2007: 22). In Ergänzung zur pragmatisch bestimmten Konzeption von Lesekompetenz, rücken hier auch Dimensionen in den Blick, die mit der Persönlichkeitsbildung verbunden sind, so etwa "ästhetische und sprachliche Sensibilität" oder "Empathiefähigkeit" (ibd.: 23). Von Interesse sind in diesem Sinn gerade literarische Texte, bei denen die Funktion der Ästhetik oder des Fremdverstehens im Zentrum stehen.

Das sozialisatorisch orientierte Modell der Lesekompetenz, welches Hurrelmann (2002) in Ergänzung zum PISA-Modell aufgestellt hat, erweitert die Dimensionen der Kognition und der Reflexion durch Merkmale, die aus der Sicht der Sozialisations- und Entwicklungsprozesse besonders wichtig sind:

- Motivation: Unter dieser Dimension werden Kompetenzen verstanden, Texte als etwas für einen selbst Bedeutungsvolles wahrzunehmen, ihnen mit positiven Erfolgserwartungen gegenüberzutreten, während der Rezeption Zielstrebigkeit und Beharrungsvermögen bzw. Persistenz zu entwickeln und aufrechtzuerhalten.

- Emotion: Unter lesespezifischen Emotionen werden Einstellungshaltungen und die Kompetenz verstanden, eigene Erfahrungen und Gefühle mit dem Textverständnis zu verbinden. Dazu gehört insbesondere beim literarischen Lesen die Kompetenz, Empathie zu Figuren und Handlungen aufzubauen, und die Kompetenz, ästhetische Konstruktionen in Texten zu genießen.

- Anschlusskommunikation: Dieser Begriff bezeichnet die Kompetenz, sich über das konstruierte Textverständnis mit anderen auszutauschen, dabei das eigene 
Textverständnis $\mathrm{zu}$ erweitern, $\mathrm{zu}$ korrigieren, den Text auf diese Weise neu zu durchdringen, bzw. die Kompetenz, Toleranz gegenüber anderen Verstehenskonstruktionen $\mathrm{zu}$ üben und die unterschiedlichen Interpretationen gemeinsam aushandeln zu können.

Diese Erweiterung des Kompetenzbegriffs ist bisher empirisch noch kaum überprüft worden. Es stellen sich in diesem Zusammenhang z. B. Fragen nach der Einheitlichkeit des Kompetenzbegriffs: Müssen wir von Teilkompetenzen ausgehen, die von einander weitgehend unabhängig sind, oder kann man von einem "Kompetenzdach" ausgehen, unter dem sich die verschiedenen Teilkompetenzen nahe bei einander befinden? Die Überprüfung der von Hurrelmann postulierten zusätzlichen Kompetenzdimensionen hat im schulischen Kontext eine lange Tradition. Der Umgang der Schule z. B. mit empathischen Aspekten des Leseverstehens ist allerdings oft stark auf normativ vorgegebene Interpretationen eingeschränkt. Dies ist einerseits auf dem Hintergrund der schwierigen Bestimmbarkeit von emotionalen Befindlichkeiten und ihren Ursachen in literarischen Texten nachvollziehbar. Andererseits widersprechen solche Deutungen genau dem selten auf Eindeutigkeit angelegten Charakter von literarischen Texten.

\subsection{MigrantInnen und Lesekompetenz}

Oben wurde bereits auf die Resultate der PISA-Studie 2000 in der Schweiz hingewiesen. Moser (2002: 117-119) belegt, dass fast 50\% der Jugendliche aus immigrierten Familien die Niveaustufe 2 nicht erreichen und als Risikogruppe eingestuft werden müssen. Allerdings weist er, wie andere Autoren auch (z. B. Rüesch 1998, Gogolin/Neumann/Roth 2003), auf den Umstand hin, dass ein großer Teil der MigrantInnen aus eher tiefen sozialen Schichten stammen, dass also ein Teil der Leseschwäche gar nicht mit der sprachlichen, sondern mit der sozialen Situation zu tun hat. In Wirklichkeit ist ein großer Anteil dieser Kategorisierung also konfundiert mit dem Sozialstatus. Der Risikoanteil der Mehrsprachigkeit wird bei statistischer Kontrolle der sozialen Schicht deshalb wesentlich reduziert. Für die Beurteilung der Auswirkungen von Mehrsprachigkeit auf Lesekompetenz ist also die soziale Platzierung innerhalb dieser Gruppe unerlässlich. Diese Erkenntnis ist in besonderem Masse relevant, wenn ein Konzept wie Resilienz fokussiert wird. Oft wird Mehrsprachigkeit an sich als ein gravierender Risikofaktor dargestellt.

\subsection{Die Lesekompetenz in der vorliegenden Studie}

Die wissenschaftliche Erhebung von Empathiefähigkeit beim Lesen von literarischen Texten ist, wie in Kapitel 8.1 dargestellt, ein schwieriges Unterfangen. Im Rahmen unseres Projekts setzten wir zur Messung der empathischen Leseleistung den am Zentrum Lesen der PH der Fachhochschule Nordwestschweiz entwickelten Test zur Erhebung der empathischen Leseleistung (TELL) ein (cf. Bertschi-Kaufmann/Schneider 2006). Textgrundlage ist eine längere Passage aus einem Jugendbuch, zu dem offene Fragen gestellt werden, die sich um das emotionale Befinden von Figuren, um Motive und um Beziehungen zwischen Figuren drehen. Die verbalen Antworten werden nach einem von Linguisten und Literaturwissenschaftlerinnen entwickelten Schema als richtig oder falsch geratet. ${ }^{16}$

Als eher kognitiv orientierter Leseverstehenstest wurde "Ein Leseverstehenstest für Erst- bis Sechstklässler (ELFE 1-6)" (Lenhard/Schneider 2006) eingesetzt. Allerdings passten wir den Test insofern an die Altersklasse der Fünfzehnjährigen an, als wir die einfachsten Items wegliessen und zwei schwierigere hinzufügten.

${ }^{16}$ Zur Interrater-Reliabilität siehe Bertschi-Kaufmann/Schneider (2006: 411). 
Mit dem Einsatz dieser beiden Erhebungsinstrumente konnten wir einen Teil des von Hurrelmann geforderten erweiterten Kompetenzbegriffs empirisch abbilden. Frühere Studien haben bereits gezeigt, dass ELFE und TELL zwar hochsignifikant aber nicht überaus stark korrelieren. Wer also im ELFE-Test gute Werte erzielt, braucht nicht unbedingt auch im TELL gut abzuschneiden. In Bezug auf die Bestimmung von Erfolg bringt eine solche Differenzierung von Teilkompetenzen deshalb auch Erfolgsdifferenzierungen mit sich.

Im Kapitel 8.2 wurde betont, dass der eigentliche Risikofaktor für die Leseleistung nicht der Migrationsstatus, sondern der Sozialstatus ist. In der vorliegenden Studie wurde der Sozialstatus operationalisiert als Mischindex aus dem elterlichen Berufsprestige (ISEI-Wert, cf. Meier 2003), dem elterlichen Bildungsabschluss und der Anzahl Bücher im Haushalt (als Teil des lesebezogenen kulturellen Kapitals der Familie). Einer der wichtigsten Prädiktoren für die Leseleistung ist die (nonverbale) Intelligenz (Artelt et al. 2001: 129). Diese Variable wurde mit dem CFT20 (Weiss 1998) gemessen.

\subsection{Leseleistungen: Mehrsprachige und Einsprachige im Vergleich}

Im letzten Kapitel wurde betont, dass die Ausdifferenzierung von Teilkompetenzen des Leseverstehens $\mathrm{zu}$ einem uneinheitlichen Erfolgsbegriff führt. Im Rahmen einer Resilienzstudie hat dies zugleich zur Folge, dass verschiedene parallele "Resilienzen" angenommen werden müssen, denn eine Person kann sich bezüglich des kognitiven Textverstehens positiv entwickeln und als resilient gelten, bezüglich des empathischen Leseleistung aber nicht. In der Folge werden immer beide Teilkompetenzen separat ausgewiesen.

Wir wollen in einem ersten Schritt die Leseleistungen der Mehrsprachigen mit denen der Einsprachigen vergleichen (a). Dabei wird sichtbar, dass - wie oben bereits aus anderen Studien berichtet - die Mehrsprachigkeit an sich wenig Effekte auf die Testresultate hat, dass vielmehr die soziale Herkunft das erklärende Element ist.

In einem zweiten Schritt wollen wir deshalb aufzeigen, welche Rolle der Sozialstatus bei der Lösung von Testaufgaben zu kognitiven Teilkompetenzen auf der einen Seite und zu empathischen Teilkompetenzen andererseits spielt (b). Schließlich widmen wir uns der Frage, auf welche Weise denn Mehrsprachige allfällige sprachliche Defizite ausgleichen (c). Dabei spielt die Grundintelligenz als Schutzfaktor eine wichtige Rolle.

(a) Wie gut ausgebildet ist das Leseverstehen der Mehrsprachigen verglichen mit den Einsprachigen?

Vergleichen wir dazu die Gruppenmittelwerte für die beiden Tests:

\begin{tabular}{|l|l|l|l|l|l|l|}
\hline & $\mathrm{n}$ & ELFE & ELFE korr. & $\mathrm{n}$ & TELL & TELL korr. \\
\hline Einsprachige & 1042 & $\begin{array}{l}0.13 \\
\text { SD }=0.96\end{array}$ & $\begin{array}{l}0.08 \\
\text { SD }=0.1\end{array}$ & 1043 & $\begin{array}{l}0.1 \\
\text { SD }=0.1\end{array}$ & $\begin{array}{l}0.06 \\
\text { SD }=1\end{array}$ \\
\hline Mehrsprachige & 452 & -0.29 & -0.18 & 456 & -0.22 & -1.4 \\
\hline F-Wert & & $60.84 * * *$ & $23.85 * * *$ & & 33.67 & $13.72^{* * *}$ \\
\hline Eta $^{2}$ & & 0.039 & 0.016 & & 0.022 & 0.009 \\
\hline
\end{tabular}

Tab. 2: Mittelwertvergleiche der Gruppe der Mehrsprachigen mit der Gruppe der Einsprachigen mit einer einfaktoriellen Varianzanalyse bezüglich ELFE und TELL bzw. bezüglich den nach Sozialstatus korrigierten Werten ELFE korr. und TELL korr. Es sind durchwegs standardisierte Werte verwendet.

Die Tabelle 2 stellt Mittelwertunterschiede zwischen den Ein- und den Mehrsprachigen bezüglich ELFE und TELL dar. Obwohl die Gruppenunterschiede (zu Gunsten der Einsprachigen) hochsignifikant sind, zeigen, liegt die Effektstärke $\left(\mathrm{Eta}^{2}\right)$ im eher geringen 
Bereich. Die Unterschiede sind zwar mit großer Sichherheit vorhanden, aber nur schwach ausgeprägt.

(b) Kontrolliert man statistisch nun noch den Sozialstatus, so sind zwar immer noch hochsignifikante Unterschiede vorhanden, aber sie sind wesentlich kleiner geworden und können vernachlässigt werden. Wir können auf diese Art nachweisen, dass Mehrsprachigkeit an sich kein Risiko ist, höchstens gepaart mit tiefem Sozialstatus kann ansatzweise von einer Risikokonstellation gesprochen werden. Interessant ist ferner, dass die Unterschiede im kognitiv orientierten ELFE-Test stärker ausgeprägt sind als beim Empathietest TELL. Auf der Ebene der Empathieleistung beim Lesen kann die Kombination von Mehrsprachigkeit und tiefem Sozialstatus nicht als Risikokonstellation bezeichnet werden. Im Hinblick auf die Schulpraxis stellt sich die Frage, wie stark die Dimension des Emotionalen beim Lesen für die Bewertung von Leseleistung berücksichtigt wird. Je mehr Gewicht der emotionalen Nähe beim Lesen beigemessen wird, desto eher verschwinden die Unterschiede zwischen Ein- und Mehrsprachigen.

(c) Die Rolle der Grundintelligenz bei der Leistung in ELFE und TELL: Mehrsprachige, das ist eine seit langem bekannte Tatsache (Grosjean 1982, 1996), weisen in den Kompetenzen der einzelnen Sprachen gegenüber Monolingualen im Durchschnitt leichte Defizite auf. Wir gehen davon aus, dass dies auch in unserer Stichprobe so ist und stellen uns die Frage, inwiefern die Grundintelligenz der Mehrsprachigen solche sprachlichen Defizite bei der Bearbeitung der Testaufgaben ausgleicht. Oder anders formuliert: Wenn Mehrsprachigkeit ein kleines Risiko darstellt, kann die Grundintelligenz als Schutzfaktor bezeichnet werden?

\begin{tabular}{|l|l|l|l|l|}
\hline & $\mathrm{n}$ & Regression ELFE-IQ: $\mathrm{R}^{2}$ & $\mathrm{n}$ & Regression TELL-IQ: $\mathrm{R}^{2}$ \\
\hline Einsprachige & 1038 & 0.051 & 1037 & 0.032 \\
\hline Mehrsprachige & 449 & 0.176 & 452 & 0.098 \\
\hline
\end{tabular}

Tab. 3: Die durch die Grundintelligenz erklärte Varianz $\left(\mathrm{R}^{2}\right)$ an ELFE bzw. TELL.

Die Resultate von Regressionsanalyse (cf. Tab. 3) zeigen, dass der Einfluss der Grundintelligenz für Mehrsprachige sowohl bei der kognitiven als auch bei der emotionalen Leseleistung ungefähr dreimal so stark ist wie bei den Einsprachigen. Zudem wird ersichtlich dass für beide Gruppen die Grundintelligenz bestimmender für die Leistung im kognitiven Bereich als im emotionalen Bereich ist.

Mehrsprachigkeit, so das Fazit aus unseren Untersuchungen zur Leseleistung, ist für sich genommen kaum ein Risiko. Hingegen ist die Kombination von Mehrsprachigkeit und tiefem Sozialstatus tatsächlich ein leichter Problembereich. Erfolgreiche mehrsprachige Jugendliche gleichen in höherem Masse als einsprachige, ihre spezifischen sprachlichen Defizite durch eine höhere Grundintelligenz aus.

\section{$9 \quad$ Fazit}

Ist Mehrsprachigkeit ein Risikofaktor, insbesondere was den Erwerb des (standardsprachlichen) Lesens und Schreibens in einer Diglossiesituation betrifft?

Die Antwort auf diese Fragen ist mehrschichtig und bedarf einiger Differenzierungen. Zunächst ist zu fragen: Risikofaktor bezüglich was? Möglicherweise und nach allgemeiner Auffassung können schriftliche Schülertexte unter dem Aspekt der Rechtschreibung und der Grammatik so analysiert werden, dass Mehrsprachigen, welche erst kurz eine deutschsprachige Schule besuchen und Deutsch als Zweitsprache zu lesen und schreiben lernen, Defizite aufgezeigt werden. In einem späteren Lernalter sind in unserer Stichprobe diesbezüglich kaum mehr Unterschiede festzustellen. Nimmt man allerdings pragmatischfunktionale Dimensionen des Schreibens als Bewertungsnorm, dann lassen sich bereits in 
einem jüngeren Lernalter, erst recht aber später kaum Unterschiede zwischen Texten von Einund Mehrsprachigen ausmachen.

Im Anschluss an diesen Befund stellt sich natürlich die Frage, welche Norm denn die gültige sein solle. Wir plädieren in unserem Beitrag nicht für die Abschaffung von Rechtschreibung und Grammatik als Bewertungsmassstab für schriftliche Texte. Wir sind aber der Ansicht, dass diese Normen ergänzt (und dadurch in ihrem Gewicht vermindert) werden sollten durch allgemeine Normen der Interaktion und deren sprachlicher Umsetzung, die für das alltägliche Schreiben, wie es im beruflichen Alltag vieler Menschen vorherrscht, maßgeblich sind.

Die Frage nach der relevanten Norm stellt sich auch im Fall des Lesens. Auch hier sprechen wir uns dafür aus, die einseitige Norm des kognitiven Textverstehens zu ergänzen durch Dimensionen, die für die Entwicklung des Lesens von großer Bedeutung sind: emotionale Nähe und kommunikativer Austausch über das Gelesene. Unsere Daten zeigen, dass das kognitive Textverstehen von Mehrsprachigen, wenn die soziale Schicht statistisch kontrolliert wird, nur wenig schwächer ausgeprägt ist als dasjenige der Einsprachigen. Noch geringfügiger wird der Unterschied, wenn die Empathieleistung beim Lesen erhoben wird.

Selbstverständlich muss in der obigen Argumentation mitbedacht werden, dass in vielen Berufen hohe Anforderungen an die literalen Kompetenzen gestellt werden. Nur indem man die Anforderungen anders definiert, wird man Jugendliche nicht auf solche Berufe vorbereiten. Wir sind allerdings der Auffassung, dass bei der Beurteilung literaler Leistungen im schulischen Unterricht die für die literale Sozialisation wichtige Dimension der als sinnvoll erlebten Einbettung von Schriftlichkeit im eigenen Leben nicht vernachlässigt werden darf, weil sonst, gerade bei risikobehafteten Jugendlichen, die Motivation für das Lesen und Schreiben gefährdet wird. Rechtschreibung und Grammatik sind nicht das Ziel eines schriftlichen Textes, sondern allenfalls Mittel zum Zweck. Und: Lesen geschieht nur ausnahmsweise mit dem Ziel, knifflige Fragen über das Gelesene zu beantworten, in vielen Lesesituationen stehen ganz andere Motive im Vordergrund. Diese darf die Schule nicht ausblenden, wenn sie bei den SchülerInnen eine habituelle Lesepraxis aufbauen will. Und dass gelesen und geschrieben wird, ist enorm wichtig, denn Lesen und Schreiben lernt man ein gutes Stück weit durch Lesen und Schreiben.

\section{Literaturangaben}

Artelt, Cordula et al. (2001): "Lesekompetenz. Testkonzeption und Ergebnisse". In: Deutsches PISA-Konsortium (ed.): PISA 2000. Basiskompetenzen von Schülerinnen und Schülern im internationalen Vergleich. Opladen: 69-137.

Artelt, Cordula et al. (2004): "Die PISA-Studie zur Lesekompetenz. Überblick und weiterführende Analysen." In: Schiefele, Ulrich/Artelt, Cordula/Schneider, Wolfgang/ Stanat, Petra (ed.): Struktur, Entwicklung und Förderung von Lesekompetenz. Vertiefende Analysen im Rahmen von PISA 2000. Wiesbaden: 139-168.

Barton, David (1994): An Introduction to the Ecology of Written Language. Oxford.

Bertschi-Kaufmann, Andrea/Schneider, Hansjakob (2006): "Entwicklung von Lesefähigkeit. Massnahmen - Messungen - Effekte. Ergebnisse und Konsequenzen aus dem Forschungsprojekt 'Lese- und Schreibkompetenzen fördern'". Schweizerische Zeitschrift für Bildungswissenschaften 3/2006: 393-424.

Gogolin, Ingrid/Neumann, Ursula/Roth, Hans-Joachim (2003): Förderung von Kindern und Jugendlichen mit Migrationshintergrund. Bonn: Bund-Länder-Kommission für Bildungsplanung und Forschungsförderung (BLK). (= Materialien zur Bildungsplanung und zur Forschungsförderung 107). 
Annelies Häcki Buhofer/Hansjakob Schneider/Christine Beckert: Mehrsprachige Jugendliche im 69 Umgang mit Dialekt und Hochsprache in der Deutschen Schweiz

Grosjean, François (1996): "Bilingualismus und Bikulturalismus. Versuch einer Definition". In: Schneider, Hansjakob/Hollenweger, Judith (eds.): Mehrsprachigkeit und Fremdsprachigkeit. Arbeit für die Sonderpädagogik? Luzern: 161-184.

Grosjean, François (1982): Life with two languages. An introduction to bilingualism. Cambridge/Mass.

Gyger, Mathilde (2005): Projekt Standardsprache im Kindergarten (PSS). Schlussbericht. Rektorat Kindergärten Basel, Arbeitsgruppe Integration.

Gyger, Mathilde (2006): Deutsch-Standard im Kindergarten - ein Schulentwicklungsprojekt in Liestal. Erster Zwischenbericht September. Kindergarten und Primarschule, Kreisschule Spezielle Förderung. Fachhochschule Nordwestschweiz, Pädagogische Hochschule.

Gyger, Mathilde/Heckendorn-Heinimann, Brigitte (1998): "Die schulische Integration fremdsprachiger Kinder und Jugendlicher in der Nordwestschweiz. Projektbericht". In: Dies. (ed.): Erfolgreich integriert? Fremd- und mehrsprachige Kinder und Jugendliche in der Schweiz. Bern: 71-143.

Buhofer, Annelies (1983): "Das alltägliche Schreiben im Berufsleben. Eine Analyse am Beispiel eines Industriebetriebs in der deutschen Schweiz". In: Grosse, Siegfried (ed.): Schriftsprachlichkeit. Düsseldorf: 137-178. (= Sprache der Gegenwart 59).

Häcki Buhofer, Annelies (1989): "Zum alltäglichen Sprachbewusstsein von Erwachsenen". OBST 40 (1989): 161-175.

Häcki Buhofer, Annelies (1995): "Soziale und kulturelle Komponenten der Schriftlichkeit. Funktionen des alltäglichen schriftlichen Ausdrucks". Babylonia 3/12: 11-15.

Häcki Buhofer, Annelies (2007): "Leseförderung in der Schweiz". Schweizer Monatshefte Nr. 03/04: 18-20.

Häcki Buhofer, Annelies/Burger, Harald (1998): Wie Deutschschweizer Kinder Hochdeutsch lernen. Stuttgart.

Häcki Buhofer, Annelies et al. (1994): "Früher Hochspracherwerb in der deutschen Schweiz: Der weitgehend ungesteuerte Erwerb durch sechs- bis achtjährige Deutschschweizer Kinder". In: Burger, Harald/Häcki Buhofer, Annelies (eds.): Spracherwerb im Spannungsfeld von Dialekt und Hochsprache. Bern: 147-198. (= Zürcher Germanistische Studien 38).

Hurrelmann, Bettina (2002): "Leseleistung - Lesekompetenz." Praxis Deutsch 176: 6-18.

Hurrelmann, Bettina (2007): "Modelle und Merkmale der Lesekompetenz." In: BertschiKaufmann, Andrea (ed.): Lesekompetenz, Leseleistung, Leseförderung. Grundlagen, Modelle und Materialien. Seele-Velber: 18-28.

Kassis, Wassilis/Schneider, Hansjakob (2004): "Schulische und familiale Determinanten der Lese- und Schreibsozialisation." In: Bertschi-Kaufmann, Andrea/Kassis, Wassilis/Sieber, Peter (eds.): Mediennutzung und Schriftlernen. Analysen und Ergebnisse zur literalen und medialen Sozialisation. Weinheim: 219-237.

Kronig, Winfried (2003): "Das Konstrukt des leistungsschwachen Immigrantenkindes". Zeitschrift für Erziehungswissenschaft 6/1: 126-141.

Landert, Karin (2007): Hochdeutsch im Kindergarten. Eine empirische Studie zum frühen Hochdeutscherwerb in der Deutschschweiz. Bern. (= Zürcher Germanistische Studien 62)

Lenhard, Wolfgang/Schneider, Wolfgang (2006): ELFE 1-6: Ein Leseverständnistest für Erst- bis Sechstklässler. Göttingen.

Luchtenberg, Sigrid (2002): "Mehrsprachigkeit und Deutschunterricht. Widerspruch oder Chance? $\mathrm{Zu}$ den Möglichkeiten von Language Awareness in interkultureller Deutschdidaktik." Informationen zur Deutschdidaktik (ide) 26/3: 27-46.

Lüdi, Georges (1999): L'enfant bilingue: chance ou surcharge cognitive? Université de Bâle, Suisse. (www.romsem.unibas.ch/sprachenkonzept/Annexe_8.html). 
Lüdi, Georges (2007): "Plurilinguisme et intégration d'enfants migrants". Terra Cognita 10/2007: 66-69. www.terra-cognita.ch/10/luedi.pdf, Stand 21.12.2007.

Lüdi, Georges/Py, Bernard (1986): Etre bilingue. Bern.

Meier, Urs (2003): Handbuch zur Berufsdatenbank. Neuchâtel.

Moser, Urs (2002): "Kulturelle Vielfalt in der Schule: Herausforderung und Chance". In: Bundesamt für Statistik, Konferenz der kantonalen Erziehungsdirektoren (ed.): Für das Leben gerüstet? Die Grundkompetenzen der Jugendlichen - Nationaler Bericht der Erhebung PISA 2000. Neuchâtel: 113-135.

Rüiesch, Peter (1998): Spielt die Schule eine Rolle? Schulische Bedingungen ungleicher Bildungschancen von Immigrantenkindern - eine Mehrebenenanalyse. Bern. (= Explorationen 18).

Schneider, Hansjakob (1994): "Wie verstehen Deutschschweizer Kinder hochdeutsche Wörter? Teil I: Wortverstehen". In: Hollenweger, Judith/Schneider, Hansjakob (eds.): Sprachverstehen beim Kind. Beiträge zu Grundlagen, Diagnose und Therapie. Luzern: 1534.

Schneider, Hansjakob/Bertschi-Kaufmann, Andrea (2006): "Wenn Schriftaneignung trotzdem gelingt - Ein Forschungsprojekt zur literalen Resilienz". Leseforum Schweiz 15: 8-11.

Weiss, Rudolf H. (1998): Grundintelligenztest Skala 2: CFT 20. Göttingen. 